
\title{
28 Research Square \\ Laboratory Predictors for COVID-19 ICU Admissions in a Caribbean Territory.
}

Chavin Gopaul ( $\sim$ Chavin.gopaul@gmail.com )

North Central Regional Health Authority

Dale Ventour

The University of West Indies St. Augustine

Davlin Thomas

North Central Regional Health Authority

\section{Research Article}

Keywords: Caribbean territory, COVID-19, biomarkers, ICU

Posted Date: November 6th, 2020

DOl: https://doi.org/10.21203/rs.3.rs-103394/v1

License: (c) (1) This work is licensed under a Creative Commons Attribution 4.0 International License.

Read Full License 


\section{Abstract}

Introduction. Understanding the symptoms associated with COVID-19 cases that require intensive care unit (ICU) attention is important in management of the life threatening case of the disease.

Method : This study was carried out with the aim of determining the laboratory indicators of ICU admission. Data from 126 COVID-19 patients (ICU patients, $n=18$, Non-ICU, $n=98$ ) were analyzed from a Caribbean Territory.

Results: The median age for the non-ICU patients was $59.0($ IQR $=23.5)$ years while the ICU patients had a median age of $62.5(\mathrm{IQR}=17.5)$. The univariate analysis indicted that laboratory indicators that were significantly associated with ICU admission included WBC $(P=0.037)$, lymphocyte $(P=0.016), L D H(P=$ 0.002), AST $(P=0.005)$ and CRP $(P=0.0001)$. However, multivariate analysis that included WBC, neutrophil, lymphocyte, PLT, AST, LDH, ALT, and CRP indicated that only AST was associated with high odds of patients being admitted to ICU (OR .002, 95\% Cl 0.000-.004, $P=0.017)$. Statistically significant area under the curve (AUC) were obtained for Neutrophil (AUC = 0.704, $P=0.007), C R P(A U C=0.81, p=$ $0.00)$ and $L D H(A U C=0.766, P=0.00)$ and $A S T(A U C=0.729, P=0.003)$.

Conclusion: The findings indicate that neutrophils, AST and LDH's ROC curves are good tests while CRP curve is a very good test. However, lymphocyte curve is a poor test for determining COVID-19 patient for ICU admission. Neutrophil, AST, LDH and CRP are suitable predictors of COVID-19 patients that should receive ICU care

\section{Introduction}

COVID-19, also known as SARS-CoV-2, was declared a pandemic by the World Health Organization (WHO) in March 2020 after Wuhan confirmed its first case in December 2019, thereafter, spreading to over 180 countries by March 2020. COVID-19 is a beta-coronavirus sharing phylogenic similarities to SARS-CoV (1). Sequencing analysis also showed that the COVID-19 virus serology was homologous to a SARS-like coronavirus(2). SARS-CoV is spread by bats, and by extension, COVID-19 is considered to be zoonotic in nature. COVID-19 has emerged as the third novel coronavirus in the last eighteen (18) years (3) and differs from the other coronaviruses in its class due to its longer incubation period and lower fatality rates (2). These novel characteristics have been thought to be the underlying factors in the rapid spread of the disease. A longer incubation time means that infected persons with the disease may have been in contact with many persons even before the onset of COVID-19 related symptoms. This poses a great challenge in the containment of the virus. Measures have been put in place worldwide to help combat the spread of the disease. These measures include washing of the hands, coughing or sneezing into the elbow, and social distancing, which have been fully implemented globally.

Due to the large spread of the virus, research has been launched into the investigation of possible laboratory predictors for COVID-19. Laboratory parameters have been used previously to shed light on the disease severity, defining the prognosis, aid in follow ups, guiding treatment and for therapeutic 
monitoring (4). Parameters such as interleukin-6 (IL-6), D-Dimer, glucose (GLU), thrombin time (TT), fibrinogen (FIB) and C-reactive protein (CRP) has shown to be indicative of patients being classified as severe or mild with COVID -19 $(5,6)$. Limited research has been done on the evaluation of the accuracy of laboratory predictors for COVID-19 patients who have been tested positive via RT-PCR.

Patients testing positive for COVID-19 are subjected to a Complete Blood Count (CBC) which serves to guide clinicians in caring for the overall health of patients. Any irregularities point to a series of health issues such as anemia, leukemia and much more. In light of the COVID-19 findings, CBC results were used to show the differentiation between patients who had a severe case of the disease and those that need to be transferred into the Intensive Care Unit (ICU) (7). Patients that had higher blood leukocyte count, $>10 * 10^{\wedge} 9 / \mathrm{L}$, were more likely to have severe COVID-19 and were admitted into ICU.

White blood cells (WBCs) are produced in the bone marrow and serves to fight against infections in the body. COVID-19 patients showed a normal or decrease in WBC and lymphocyte counts at admission into the hospital (8-10). Even though COVID-19 survivors and non-survivors had normal WBCs in Zhao et al., 2020 study, the non-survivors had higher WBC count and slightly reduced lymphocyte counts (10).

Exaggerated elevation of inflammatory cytokines in the body, such as Interleukin-6 (IL-6), causes the onset of a cytokine storm, as seen in COVID-19 patients, which results in multiple organ failure (MOF) and Acute Respiratory Distress Syndrome (ARDS) $(9,11-14)$. Rapid viral replication in the body causes vigorous pro-inflammatory response causing apoptosis in the lung's epithelial tissue, leading to hypoxia and $\operatorname{ARDS}(12,15)$.

Studies, such as Zhang L. et al., 2020, reported elevated levels of D-dimer being one of the more common laboratory finding in COVID-19 patients requiring hospitalization $(6,16)$. D-dimer elevation was associated with a hypercoagulable state of patients, however, its specificity on the main cause of elevation may not be known as D-dimer elevations were associated with several unfavorable events such as occlusion, sepsis, micro-thrombosis, and intravascular coagulation $(7,16,17)$.

Lactate Dehydrogenase (LDH) is an enzyme expressed in all cells such as the heart, liver, muscle, kidney, lung and bone marrow (2). During biochemistry laboratory tests, increased LDH levels signaled that there is damage to the cells that it is normally expressed in. Elevated levels of LDH has been associated with worse clinical outcome of COVID-19 patients $(7,18)$. Monitoring both the LDH and lymphocyte count can differentiate between ICU and non-ICU patients.

Liver damage was reported in SARS-CoV patients which led researchers to investigate the effect of COVID-19 on the liver (19). Several studies reported similar liver abnormalities in COVID-19 patients, both at admission to the hospital and during their hospital stay $(6,19)$. Elevated levels of alanine aminotransferase (ALT) and aspartate aminotransferase (AST) were reported, ranging from $14 \%$ to $53 \%$ $(9,19,20)$. ALT, AST, Total Bilirubin, Creatinine, and Blood Urea Nitrogen (BUN) were within normal ranges according to Ruoqing Li et al., 2020 (8), however, these results were taken at admission into the hospital as opposed to during hospitalization. 
C-Reactive Proteins (CRP) are produced in the liver and studies have shown highly elevated levels in COVID-19 patients which is also a key indicator of MAS $(8-10,13)$. Higher levels of CRP has been associated with lung lesions and are very important in assessing the severity of the disease in the patient (21). Excessive inflammation was also reflected by very high CRP levels in patients with severe disease state (7).

Laboratory predictors are key components in providing faster and more accurate diagnosis of the novel COVID-19. This is tremendously important due to the rapid transmission of the virus from person to person and it's long incubation time. Early and accurate diagnosis of COVID-19 can reduce the load on the health care systems worldwide.

\section{Methods}

For this retrospective study, data from one hundred and thirteen (113) patients with confirmed 2019nCoV, managed in a Caribbean Territory from March $12^{\text {th }} 2020$ to April $12^{\text {th }} 2020$, were analyzed. Cases were confirmed by an accredited laboratory utilising real time reverse transcription polymerase chain reaction assay (RT-PCR). Clinical Data, demographics and clinical symptomology were recorded. Ethical approval was granted from the of the North Central Regional Health Authority.

\section{Statistical Analysis}

All statistical analysis was processed using IBM SPSS 22.0 statistical software. Descriptive statistics that included median and interquartile range, and frequencies were used in the analysis of the characteristics of the studied patients. Means and standard deviation were used to describe the laboratory test outcome. Univariate and multivariate analyses were used to determine the presence of statistically significant association between laboratory indicators and ICU admission. Receiver operating characteristic (ROC) curve and AUC were used to analyze the optimal cut-off for prediction ICU admission. AUC 0.9 to 1 was defined as excellent accuracy, 0.8 to 0.9 as very good, 0.7 to 0.8 as good, 0.6 to 0.7 as sufficient, 0.5 to 0.6 as bad, and $<0.5$ as poor (useless test). A P value $<.05$ denoted statistical significance.

\section{Results}

\section{Characteristics of the studied patients}

Among the patients who took part in the study 45 (38.8 \%) were males while 71 (61.2\%) were females. The number of male and female patients differed significantly between the ICU and non-ICU patients ( $p=$ 0.008 ) with $66.7 \%$ of the ICU patients being male while among non-ICU patients $66.3 \%$ were females. The median age for the non-ICU patients was $59.0(I Q R=23.5)$ years while the ICU patients had a median age of $62.5(I Q R=17.5)$. The observed age difference between the ICU and non-ICU patients was not statistically significant $(P=0.101)$. 


\section{Laboratory test outcome}

The biochemistry laboratory findings in Table 1 indicate that the AST level for the non-ICU patients (M = $45.4, \pm 39.84)$ was lower compared to the ICU patients $(80.6 \pm 5.76)$. The reported difference was statistically significant $(P=0.005)$. Table 1 also indicates that LDH was statistically significantly higher among ICU patients $(994.2, \pm 423.19)$ compared to non-ICU patients $(669.6 \pm 40.74, P=0.002)$. The CRP concentration outcome is the other biochemistry test outcome that was statistically significantly different between ICU $(93.2, \pm 20.09)$ and non-ICU patients $(35.8, \pm 5.33)$. However, the findings presented in Table 1 indicate that the concentration of ALT did not vary between the ICU and non- ICU patients $(P=0.76)$. The $\mathrm{CBC}$ findings in Table 1 indicate that the concentration of WBC was significantly higher among the ICU patients $(8.26, \pm 3.61)$ compared to the non-ICU patients $(6.7, \pm 2.47, \mathrm{P}=0.037)$. The findings also indicate that concentration of the lymphocytes were statistically higher among the non-ICU patients (2.05 $\pm 0.75)$ compared to the ICU patients $(1.57 \pm 0.71, P<0.016)$. The level of neutrophil in the non-ICU $(6.11$ $\pm 18.79)$ did not statistically differ from the ICU $(6.03 \pm 3.41 \mathrm{P}=0.986)$

Table 1: Demographic and laboratory parameters among the ICU and Non-ICU COVID-19 patients

\begin{tabular}{|c|c|c|c|c|c|}
\hline \multirow[t]{2}{*}{ Variables } & \multirow[t]{2}{*}{ Normal Range } & Non ICU(n=) & $\operatorname{ICU}(n=)$ & \multirow{2}{*}{$\begin{array}{l}\text { Chi } \\
\text { squared }\end{array}$} & \multirow{2}{*}{$\begin{array}{l}\mathrm{P} \\
\text { value }\end{array}$} \\
\hline & & Number (\%) & Number (\%) & & \\
\hline Age Median(IQR), years & & $59.0(23.5)$ & $62.5(17.5)$ & & 0.101 \\
\hline \multicolumn{6}{|l|}{ Sex,n(\%) } \\
\hline Male & & 33 (33.7) & $12(66.7)$ & \multirow[t]{2}{*}{6.972} & \multirow[t]{2}{*}{0.008} \\
\hline Female & & $65(66.3)$ & $6(33.3)$ & & \\
\hline LDH(U/L) & $140-280$ & $\begin{array}{l}669.6 \\
(40.74)\end{array}$ & $\begin{array}{l}994.2 \\
(423.19\end{array}$ & & 0.002 \\
\hline $\operatorname{ALT}(\mathrm{U} / \mathrm{L})$ & $7-55$ & $58.6(9.54)$ & $52.0(9.93)$ & & 0.76 \\
\hline AST(U/L) & $8-48$ & $\begin{array}{l}45.4 \\
(39.84)\end{array}$ & $80.6(75.76)$ & & 0.005 \\
\hline CRP(U/L) & $<10 \mathrm{mg} / \mathrm{L}$ & $35.8(5.33)$ & $93.2(20.09)$ & & 0.00 \\
\hline $\begin{array}{l}\text { Lymphocyte count } x \\
\text { 1000/UL }\end{array}$ & $1-4$ & $2.05(0.75)$ & $1.57(0.71)$ & & 0.016 \\
\hline $\begin{array}{l}\text { Neutrophil count } x \\
\text { 1000/UL }\end{array}$ & $2.5-8$ & $6.11(18.79)$ & $6.03(3.41)$ & & 0.986 \\
\hline WBC count $x$ 1000/UL & $\begin{array}{l}4.5-11.0 \times 10^{9} \\
/ L\end{array}$ & $6.7(2.47)$ & $8.26(3.61)$ & & 0.037 \\
\hline
\end{tabular}


The univariate analysis indicted that laboratory indicators that were significantly associated with ICU admission included WBC $(P=0.037)$, lymphocyte $(P=0.016), \operatorname{LDH}(P=0.002)$, AST $(P=0.005)$ and CRP $(P=0.0001)$. However, multivariate analysis that included WBC, neutrophil, lymphocyte, PLT, AST, LDH, ALT, and CRP indicated that only AST was associated with high odds of patients being admitted to ICU (OR .002, 95\% Cl (0.001-0.004), $\mathrm{P}=0.017$ ) see Table 2 .

Table 2: Univariate and multivariate analyses for predictors of ICU admission among COVID-19 patients.

\begin{tabular}{|lllll|}
\hline & Univariate analysis & \multicolumn{3}{c|}{ Multivariate analysis } \\
\hline & Odds ratio $(95 \% \mathrm{Cl})$ & $\mathrm{P}$ & Odds ratio $(95 \% \mathrm{Cl})$ & $\mathrm{P}$ \\
\hline WBC & $-0.027(-0.233-0.179)$ & 0.037 & $.031(-.001-.064)$ & .060 \\
\hline Neutrophil & $0.00(-0.005-0.004)$ & 0.986 & $-.001(-.006-.003)$ & .520 \\
\hline Lymphocyte & $-0.12(-0.217-0.023)$ & 0.016 & $-.059(-.172-.054)$ & .302 \\
\hline PLT & $0.00(-0.001-0.001)$ & 0.589 & $000(-.001-.000)$ & .304 \\
AST & $0.002(0.001-0.004)$ & 0.005 & $.002(.000-.004)$ & .017 \\
\hline LDH & $0.00(0.00-0.00)$ & 0.002 & $.000(.000-.000)$ & .346 \\
\hline ALT & $0.00(-0.001-0.001)$ & 0.076 & $-.001(-.002-.000)$ & .267 \\
\hline CRP & $0.002(0.001-0.003)$ & 0.000 & $.001(-.001-.002)$ & .505 \\
\hline
\end{tabular}

Table 3, Figure 1 and Figure 2 show the area under the ROC curve of the assessed biochemistry parameters (Figure 1) and the $\mathrm{CBC}$ parameters (Figure 2). The biochemistry with good accuracy was Neutrophil $(A \cup C=0.704, P=0.007)$ while lymphocyte AUC $(0.309)$ indicates that the parameter has no value in the prediction of ICU patients. The findings presented in Table 3 indicate that the AUC for WBC was not statistically significant. For the CBC parameters, Table 3 shows that statistically significant AUC $s$ were obtained for CRP (AUC $=0.81, p=0.00)$ and LDH $(A U C=0.766, P=0.00)$ and AST (AUC=0.729, P= 0.003 ). The meaning of the statistically significant ROC curves were interpreted based on the criteria where AUC 0.9 to 1 was defined as excellent accuracy, 0.8 to 0.9 as very good, 0.7 to 0.8 as good, 0.6 to 0.7 as sufficient, 0.5 to 0.6 as bad, and less than 0.5 as poor (useless test). As shown in Table 3 neutrophils, AST and LDH's ROC curves are good tests while CRP curve is a very good test. However, lymphocyte curve is a poor test.

Table 3: Area under ROC curve for the laboratory parameters among the ICU COVID-19 patients 


\begin{tabular}{|lllll|}
\hline Parameters & AUC & $95 \% \mathrm{Cl}$ & $P$ & Interpretation \\
\hline WBC & 0.646 & $0.501-0.790$ & .053 & \\
\hline Neutrophil & 0.704 & $0.574-0.835$ & .007 & Good \\
\hline Lymphocyte & 0.309 & $0.171-0.447$ & .011 & Poor \\
\hline PLT & 0.459 & $0.297-0.621$ & .592 & \\
AST & 0.729 & $0.580-0.878$ & .003 & Good \\
LDH & 0.766 & $0.627-0.904$ & .000 & Good \\
ALT & 0.628 & $0.495-0.761$ & .091 & \\
\hline CRP & 0.810 & $0.722-0.898$ & .000 & Very good \\
\hline
\end{tabular}

\section{Discussion}

This study showed that there is a difference in various biochemical and $\mathrm{CBC}$ test parameters between ICU and non-ICU patients. The outcome showed elevated levels of AST, LDH, WBC, lymphocytes and CRP among ICU patients compared to the non-ICU patients. It is also evident from the findings of this study that the parameters that have good specificity and sensitivity in the prediction of the ICU COVID-19 patients include neutrophil, AST, LDH and CRP. The assessment of the demographic variables revealed that ICU patients are older compared to non-ICU counterparts, which corroborates findings of the previous studies (22-24). However, the age difference between the ICU and non-ICU patients was smaller compared to the age difference reported by previous researchers, which was noted to be over a 10-year difference (23). A significantly high number of male ICU patients compared to the female patients that was observed in this study support the observation by previous studies that indicated that gender of the patients is a potential determinant of the likelihood of the patients to develop serious complications that might require ICU care $(22,25,26)$.

The univariate analysis findings of the study regarding the CBC parameters contradict the outcome obtained by previous researchers $(4,27,28)$. The observed lack of statistically significant difference in the level of neutrophils among ICU and non-ICU patients contradict previous researchers who concluded that elevated neutrophils concentration is characteristic feature of patients with severe cases (28-30). Evidence suggest that severe cases of COVID-19 is associated with elevated levels of neutrophils, which increase inflammation, and haemorrhages especially in the lungs and requires the patient to be placed in intensive care $(29,30)$.

However, Mardani et al. (28) supports the observation of this study regarding the reduced concentration of WBC among ICU COVID-19 patients. The univariate assessment of the CBC parameters showed that among the ICU patients, the increase in the concentration of lymphocytes is associate with significant reduction in the odds of ICU hospitalization. This outcome therefore suggest that reduction of the 
lymphocytes increases odds of ICU hospitalization. The reduced concentration of the lymphocyte is termed as lymphopenia and has been reported to be associated with COVID-19 severity $(26,31,32)$. The study carried out by Wang et al. (23) documented the importance of lymphopenia in the identification of the COVID-19 patients that required ICU care. Fan et al. in the assessment of the baseline characteristics of the COVID-19 patients requiring ICU care also noted that the patients had significantly low levels, which further corroborates the obtained findings. Wu et al. (26) also noted that COVID-19 patients who are diagnosed with lymphopenia are likely to be experiencing respiratory distress syndrome, which according to the researchers is associated with elevated levels of neutrophils.

The biochemistry test findings of this study, which showed an elevated level of CRP, AST, LDH among ICU patients corroborates the conclusion made by Mardani et al. (33). Evidence suggest that the increased levels of AST among severe cases of COVID-19 is associated with liver damage (34-36). The findings of this study regarding the lack of difference in the level of ALT among the ICU and non-ICU patients contradict some of the previous studies $(4,37)$. However, it showed be noted that elevated levels of AST have been noted to more common compared to ALT, which explains the observation made in this study (38). The study carried out by Fan et al. (23) also noted that the COVID-19 patients that required ICU care had higher concentration of LDH. Other researchers who have reported higher concentration of LDH among ICU COVID-19 patients include $(4,39)$. Various researchers have also noted that severe cases of COVID-19 develop neutrophilia during hospitalization $(4,23)$.

However, it should be noted the multivariate analysis showed that AST is the only parameter that show significant association with ICU admission of COVID-19 patients. When the effect of WBC, neutrophil, lymphocyte, PLT, AST, LDH, ALT, and CRP is controlled, the increase in the level of AST was associated significant increase in the odd of ICU admission, which collaborate the observation made by Mardani et al. (33). The ROC curves that were obtained in this study provided information regarding the sensitivity and specificity of the various parameters in the identification of the ICU and non-ICU COVID-19 patients. It is evident from this study that the only good predictors are neutrophil, AST, LDH and CRP. The reported excellent accuracy of CRP and neutrophils in the prediction of patients with COVID-19 corroborates the findings obtained by Mardani et al. (33). However, unlike the findings of this study regarding the accuracy of LDH and AST, which was shown to be good, other researchers indicate that the test is excellent with AUC of above 0.8 (33). It should also be noted that although the current study reported ALT to be of no value in the prediction of ICU COVID-19 patients, other researchers observed that the test offered excellent prediction accuracy $(5,33,40)$. The variation in the obtained outcome could be associated with the difference in the patients and the progression of the disease in different individuals especially with respect to the integrity of the liver $(34,36)$.

The findings of this study have various practice implications in the treatment of patients with COVID-19. As indicated by previous researchers, COVID-19 severity varies across different cases $(33,36)$. It is therefore important to develop an approach through which patients with the likelihood of developing serious complications are identified and provided adequate care. The findings of this study indicate that biochemical and the CBC tests are important in the determination of whether COVID-19 patients require 
ICU care or not. According to this study, the laboratory tests that need to be prioritized in the determination of the risk of the patients developing serious COVID-19 complications that might require ICU care include AST, LDH and CRP tests. The use of CRP ROC curve is most preferred because it was noted to be very good test. The next most preferred are the AST and LDH's ROC curves, which were observed to be good tests. The use of lymphocyte curve should be avoided because it was reported to be a poor test.

It should, however, be noted that there are limitations that need to be taken into consideration in the interpretation of the findings of the study. One of the limitations relates to the sample that was used, which had unequal number of males and females. It should also be noted that the study did not control for the effect of pre-existing health conditions. It is important for future studies to determine whether the recorded observations occurred independently of the potential pre-existing health conditions such as hypertension and diabetes.

Despite the highlighted limitations, this study has provided insights into the laboratory parameters that can be used to predict the severity of COVID-19 cases. It is evident from the study that neutrophil, AST, LDH and CRP are good predictors of COVID-19 patients that should receive ICU care. It is therefore recommended that health care providers should include the use of the highlighted parameters in making an evidence-based decision regarding patient management especially where there are limited ICU facilities.

\section{Declarations}

\section{Conflict of Interests}

The authors declare that there are no conflict of interests.

\section{Author Contribution}

CDG, DV, were responsible for data analysis with intellectual contributions from DT. CDG and DV drafted the article. All authors contributed to the conception and design of the paper, interpretation of data, and critical revisions contributing to the intellectual content and approval of the final version of the manuscript.

\section{Funding}

The authors have not received any funding or benefits from industry or elsewhere to conduct this study.

\section{References}

1. Guan W-j, Ni Z-y, Hu Y, Liang W-h, Ou C-q, He J-x, et al. Clinical Characteristics of Coronavirus Disease 2019 in China. New England Journal of Medicine. 2020;382(18):1708-20. 
2. Frater JL, Zini G, d'Onofrio G, Rogers HJ. COVID-19 and the clinical hematology laboratory. Int J Lab Hematol. 2020;42 Suppl 1:11-8.

3. Azkur AK, Akdis M, Azkur D, Sokolowska M, van de Veen W, Brüggen MC, et al. Immune response to SARS-CoV-2 and mechanisms of immunopathological changes in COVID-19. Allergy. 2020.

4. Lippi G, Plebani M. Laboratory abnormalities in patients with COVID-2019 infection. Clin Chem Lab Med. 2020;58(7):1131-4.

5. Gao Y, Li T, Han M, Li X, Wu D, Xu Y, et al. Diagnostic utility of clinical laboratory data determinations for patients with the severe COVID-19. J Med Virol. 2020;92(7):791-6.

6. Lake MA. What we know so far: COVID-19 current clinical knowledge and research. Clin Med (Lond). 2020;20(2):124-7.

7. Zhang G, Zhang J, Wang B, Zhu X, Wang Q, Qiu S. Analysis of clinical characteristics and laboratory findings of 95 cases of 2019 novel coronavirus pneumonia in Wuhan, China: a retrospective analysis. Respir Res. 2020;21(1):74.

8. Li R, Tian J, Yang F, Lv L, Yu J, Sun G, et al. Clinical characteristics of 225 patients with COVID-19 in a tertiary Hospital near Wuhan, China. J Clin Virol. 2020;127:104363.

9. Chen N, Zhou M, Dong X, Qu J, Gong F, Han Y, et al. Epidemiological and clinical characteristics of 99 cases of 2019 novel coronavirus pneumonia in Wuhan, China: a descriptive study. The Lancet. 2020;395(10223):507-13.

10. Zhao Y, Nie H-X, Hu K, Wu X-J, Zhang Y-T, Wang M-M, et al. Abnormal immunity of non-survivors with COVID-19: predictors for mortality. Infectious Diseases of Poverty. 2020;9(1):108.

11. Henry BM, de Oliveira MHS, Benoit S, Plebani M, Lippi G. Hematologic, biochemical and immune biomarker abnormalities associated with severe illness and mortality in coronavirus disease 2019 (COVID-19): a meta-analysis. Clin Chem Lab Med. 2020;58(7):1021-8.

12. Ye Q, Wang B, Mao J. The pathogenesis and treatment of the 'Cytokine Storm' in COVID-19. J Infect. 2020;80(6):607-13.

13. McGonagle D, Sharif K, O'Regan A, Bridgewood C. The Role of Cytokines including Interleukin- 6 in COVID-19 induced Pneumonia and Macrophage Activation Syndrome-Like Disease. Autoimmun Rev. 2020;19(6):102537.

14. Ruan Q, Yang K, Wang W, Jiang L, Song J. Clinical predictors of mortality due to COVID-19 based on an analysis of data of 150 patients from Wuhan, China. Intensive Care Med. 2020;46(5):846-8.

15. Li X, Ma X. Acute respiratory failure in COVID-19: is it "typical" ARDS? Crit Care. 2020;24(1):198-.

16. Zhang L, Yan X, Fan Q, Liu H, Liu X, Liu Z, et al. D-dimer levels on admission to predict in-hospital mortality in patients with Covid-19. J Thromb Haemost. 2020;18(6):1324-9.

17. Connors JM, Levy JH. COVID-19 and its implications for thrombosis and anticoagulation. Blood. 2020;135(23):2033-40.

18. Henry BM, Aggarwal G, Wong J, Benoit S, Vikse J, Plebani M, et al. Lactate dehydrogenase levels predict coronavirus disease 2019 (COVID-19) severity and mortality: A pooled analysis. Am J Emerg 
Med. 2020;38(9):1722-6.

19. Chai X, Hu L, Zhang Y, Han W, Lu Z, Ke A, et al. Specific ACE2 Expression in Cholangiocytes May Cause Liver Damage After 2019-nCoV Infection. bioRxiv. 2020:2020.02.03.931766.

20. Cai Q, Huang D, Yu H, Zhu Z, Xia Z, Su Y, et al. COVID-19: Abnormal liver function tests. J Hepatol. 2020.

21. Wang L. C-reactive protein levels in the early stage of COVID-19. Med Mal Infect. 2020;50(4):332-4.

22. Jain V, Yuan JM. Predictive symptoms and comorbidities for severe COVID-19 and intensive care unit admission: a systematic review and meta-analysis. Int J Public Health. 2020;65(5):533-46.

23. Fan BE, Chong VCL, Chan SSW, Lim GH, Lim KGE, Tan GB, et al. Hematologic parameters in patients with COVID-19 infection. Am J Hematol. 2020;95(6):E131-E4.

24. Lee PI, Hu YL, Chen PY, Huang YC, Hsueh PR. Are children less susceptible to COVID-19? J Microbiol Immunol Infect. 2020;53(3):371-2.

25. Gausman J, Langer A. Sex and Gender Disparities in the COVID-19 Pandemic. J Womens Health (Larchmt). 2020;29(4):465-6.

26. Remuzzi A, Remuzzi G. COVID-19 and Italy: what next? Lancet (London, England). 2020;395(10231):1225-8.

27. Velavan TP, Meyer CG. Mild versus severe COVID-19: Laboratory markers. Int J Infect Dis. 2020;95:304-7.

28. Wang D, Hu B, Hu C, Zhu F, Liu X, Zhang J, et al. Clinical Characteristics of 138 Hospitalized Patients With 2019 Novel Coronavirus-Infected Pneumonia in Wuhan, China. JAMA. 2020;323(11):1061-9.

29. Laforge M, Elbim C, Frère C, Hémadi M, Massaad C, Nuss P, et al. Author Correction: Tissue damage from neutrophil-induced oxidative stress in COVID-19. Nat Rev Immunol. 2020;20(9):579.

30. Wu C, Chen X, Cai Y, Xia J, Zhou X, Xu S, et al. Risk Factors Associated With Acute Respiratory Distress Syndrome and Death in Patients With Coronavirus Disease 2019 Pneumonia in Wuhan, China. JAMA Intern Med. 2020;180(7):1-11.

31. Bermejo-Martin JF, Almansa R, Menéndez R, Mendez R, Kelvin DJ, Torres A. Lymphopenic community acquired pneumonia as signature of severe COVID-19 infection. J Infect. 2020;80(5):e23-e4.

32. Fathi N, Rezaei N. Lymphopenia in COVID-19: Therapeutic opportunities. Cell Biol Int. 2020;44(9):1792-7.

33. Mardani R, Ahmadi Vasmehjani A, Zali F, Gholami A, Mousavi Nasab SD, Kaghazian H, et al. Laboratory Parameters in Detection of COVID-19 Patients with Positive RT-PCR; a Diagnostic Accuracy Study. Arch Acad Emerg Med. 2020;8(1):e43.

34. Ding Y, He L, Zhang Q, Huang Z, Che X, Hou J, et al. Organ distribution of severe acute respiratory syndrome (SARS) associated coronavirus (SARS-CoV) in SARS patients: implications for pathogenesis and virus transmission pathways. J Pathol. 2004;203(2):622-30.

35. Tan YJ, Fielding BC, Goh PY, Shen S, Tan TH, Lim SG, et al. Overexpression of 7a, a protein specifically encoded by the severe acute respiratory syndrome coronavirus, induces apoptosis via a 
caspase-dependent pathway. J Virol. 2004;78(24):14043-7.

36. Xu Z, Shi L, Wang Y, Zhang J, Huang L, Zhang C, et al. Pathological findings of COVID-19 associated with acute respiratory distress syndrome. Lancet Respir Med. 2020;8(4):420-2.

37. Zhang C, Shi L, Wang FS. Liver injury in COVID-19: management and challenges. Lancet Gastroenterol Hepatol. 2020;5(5):428-30.

38. D'Antiga L. Coronaviruses and Immunosuppressed Patients: The Facts During the Third Epidemic. Liver Transpl. 2020;26(6):832-4.

39. Huang C, Wang Y, Li X, Ren L, Zhao J, Hu Y, et al. Clinical features of patients infected with 2019 novel coronavirus in Wuhan, China. Lancet. 2020;395(10223):497-506.

40. Wang Y, Liu Y, Liu L, Wang X, Luo N, Li L. Clinical Outcomes in 55 Patients With Severe Acute Respiratory Syndrome Coronavirus 2 Who Were Asymptomatic at Hospital Admission in Shenzhen, China. J Infect Dis. 2020;221(11):1770-4.

\section{Figures}

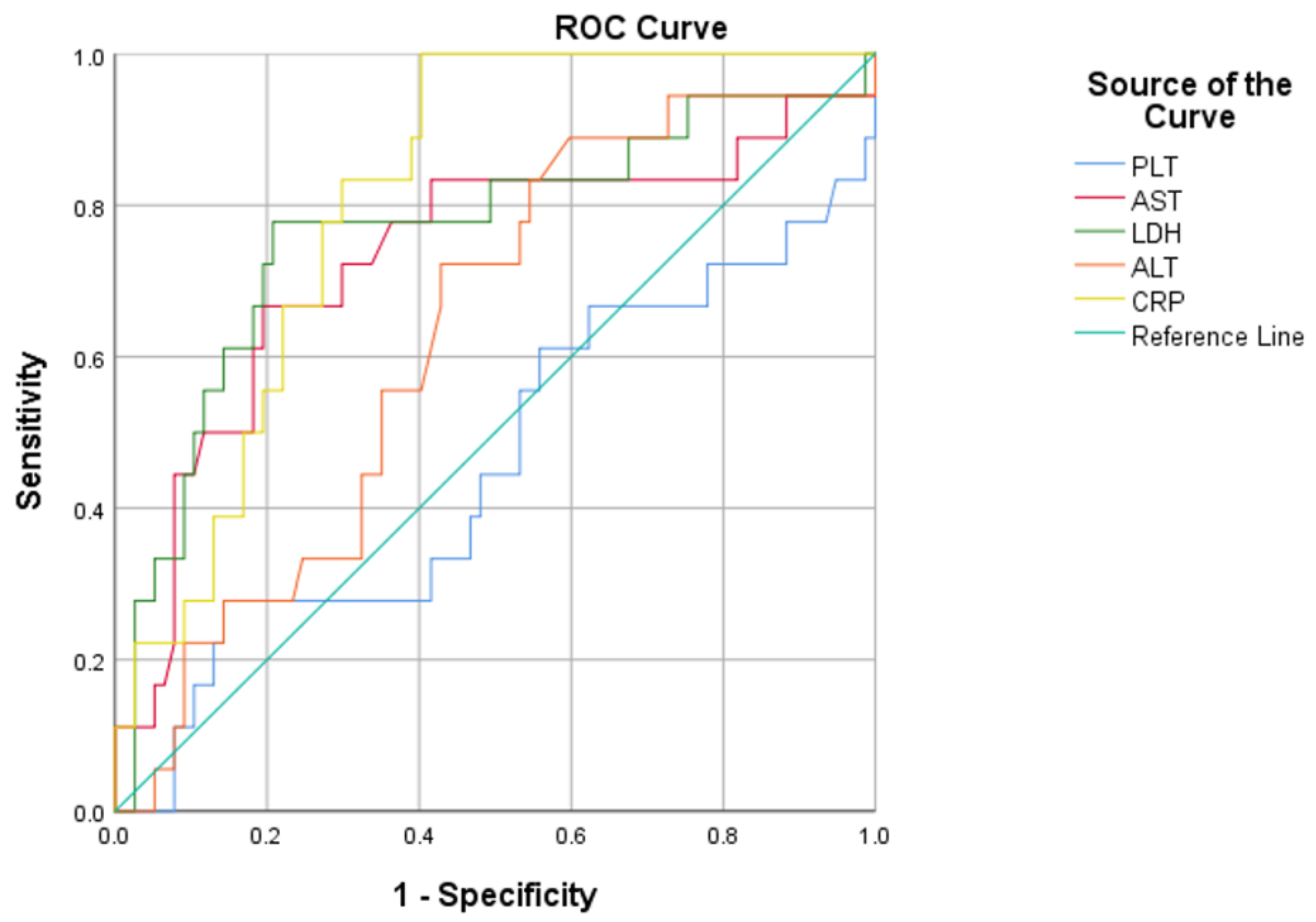

Diagonal segments are produced by ties. 
Figure 1

ROC curve for the biochemistry test outcome for the prediction of ICU patients

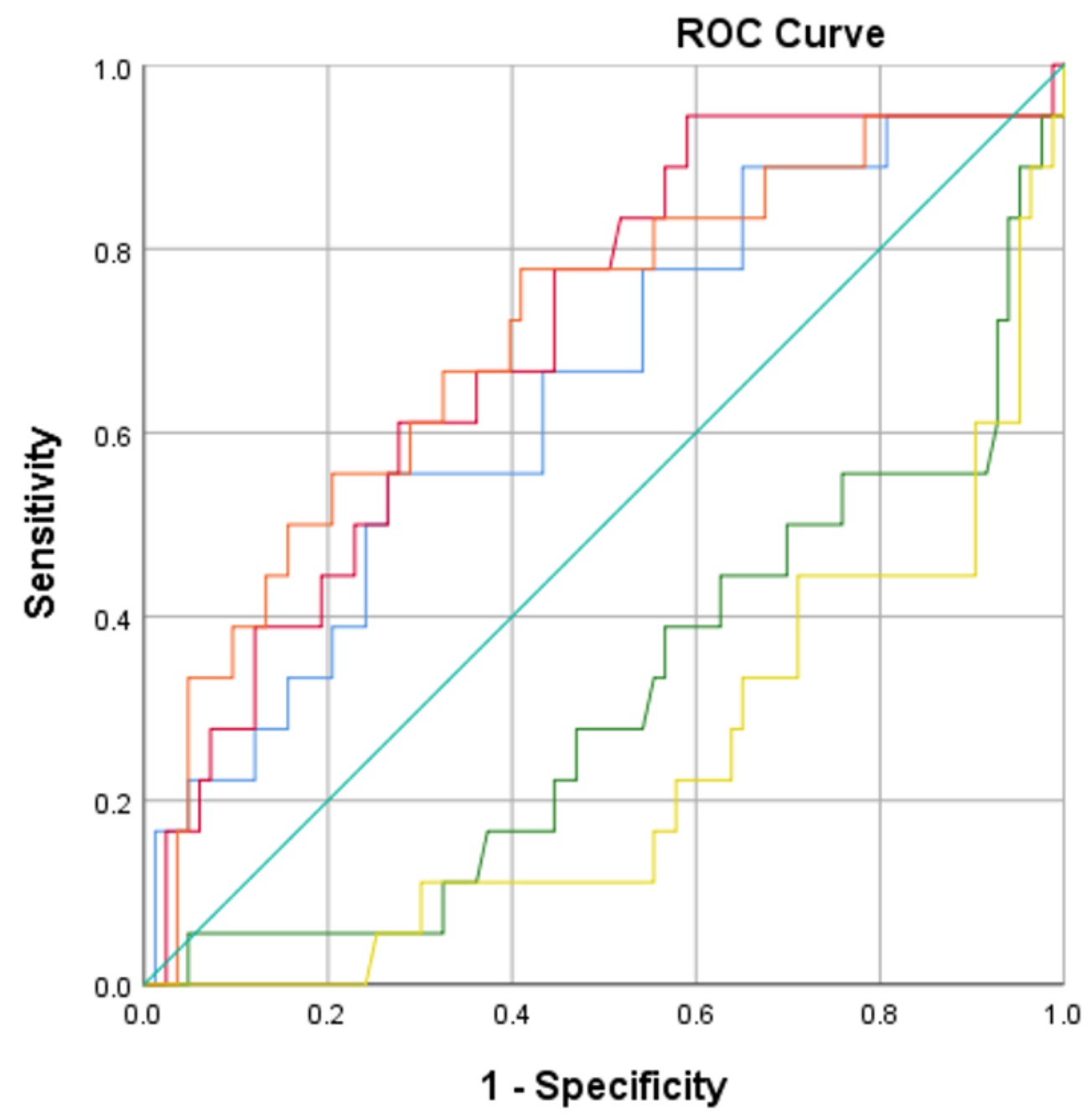

\section{Source of the} Curve

- WBC

Neutrophil Lymphocyte Neutrophil \% Lymphocyte \% Reference Line

Diagonal segments are produced by ties.

Figure 2

ROC curve for the $\mathrm{CBC}$ test outcome for the prediction of ICU patients 